\title{
The Effect of Olaparib and Bortezomib Combination Treatment on Ovarian Cancer Cell Lines
}

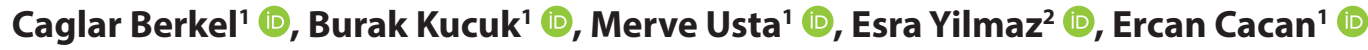 \\ 'Tokat Gaziosmanpasa University, Department of Molecular Biology and Genetics, Tokat, Turkey \\ ${ }^{2}$ Tokat Gaziosmanpasa University, Department of Biology, Tokat, Turkey
}

ORCID IDs of the authors: C.B. 0000-0003-4787-5157; B.K. 0000-0002-9325-2359; M.U. 0000-0002-5086-6270; E.Y. 0000-0002-9922-8341; E.C. 0000-0002-3487-9493

Please cite this article as: Berkel C, Kucuk B, Usta M, Yilmaz E, Cacan E. The Effect of Olaparib and Bortezomib Combination Treatment on Ovarian Cancer Cell Lines. Eur J Biol 2020; 79(2): 115-123. DOI: 10.26650/EurJBiol.2020.0035

\begin{abstract}
Objective: Ovarian cancer (OC) is the deadliest gynecologic malignancy and has a poor survival rate due to late diagnosis and chemoresistance development. In the standard treatment of OC, platinum-based chemotherapeutics are used. However, following several rounds of chemotherapy, these drugs' efficacy eventually becomes limited due to the development of chemoresistance in most patients who previously responded to this treatment. Therefore, overcoming chemoresistance in the treatment of $\mathrm{OC}$ is of high importance. In this study, we investigated the effect of combinatorial inhibition of poly(ADPribose) polymerase (PARP) and proteasome by olaparib and bortezomib on chemosensitive and chemoresistant OC cell lines.

Materials and Methods: We used sulphorhodamine B assay to screen cell viability following drug treatments alone or in combination, and used the cytotoxicity data to model the effect of drugs on cell death in R programming environment. In addition to olaparib and bortezomib, we performed cytotoxicity screenings where we applied cisplatin to OC cells. We also carried out flow cytometry analysis to quantify apoptotic cells following treatments.

Results: We showed that combination treatment was more effective on chemosensitive OC cell lines when cisplatin was not used. In the presence of cisplatin, olaparib and bortezomib combination treatment resulted in higher cytotoxicity in chemoresistant OC lines compared to chemosensitive OC cell lines. Combinatorial inhibition of PARP and proteasome led to a higher number of apoptotic cells in OV2008 chemosensitive cell line compared to drugs alone.

Conclusion: Our data shows that olaparib and bortezomib combination treatment might show promise in vivo in the treatment of OC. Also, the efficacy of this combination treatment might be dependent on OC cells' chemosensitivity profiles.

Keywords: Ovarian cancer, olaparib, bortezomib, chemoresistance, proteasome
\end{abstract}

\section{INTRODUCTION}

Ovarian cancer is the most lethal gynecologic malignancy, with an overall 5-year survival rate of less than $25 \%$ and with approximately 30,000 new cases and over 18,000 deaths worldwide each year (1). Cisplatin is used for the standard treatment of ovarian cancer as a first-line chemotherapeutic agent, but its efficacy in the clinic is limited due to resistance development (2). Most patients, initially responding to cisplatin treatment, are ultimately diagnosed with a relapse of chemoresistant disease within two years, leading to therapeutic failure
$(3,4)$. Olaparib, a poly (ADP-ribose) polymerase (PARP) inhibitor (PARPi), is approved as a maintenance treatment for platinum-sensitive relapsed ovarian cancer, regardless of its BRCA1/2 mutation status (5). PARP proteins have the ability to catalyze the transfer of ADP-ribose to its target proteins and have crucial functions in many cellular processes including transcription, replication, recombination, modulation of chromatin structure and, most importantly in this case, DNA repair and DNA damage response (6). However, PARP inhibition with olaparib treatment might result in PARPi resistance and possible mechanisms are currently being investigated (7). 
Bortezomib, a first-in-class proteasome inhibitor, is used for the treatment of adults with multiple myeloma (MM) or mantle cell lymphoma. However, its administration does not show significant therapeutic efficacy in solid tumors, including ovarian cancer (8). Inhibition of proteasome function with bortezomib leads to an accumulation of damaged proteins, resulting in the activation of caspase cascade and ultimately cell death (9). Although it is not effective in ovarian cancer as a single agent, the combination of bortezomib with olaparib may result in an increased cytotoxicity as well as a decrease of PARPi resistance. To our knowledge, to date, no previous study has reported the effect of combinatorial inhibition of proteasome and PARP with the combination of bortezomib and olaparib on ovarian cancer cell viability in vitro.

In the current study, we investigated the effect of bortezomib and olaparib combination treatment on chemoresistant and chemosensitive ovarian cancer cell lines in the presence or absence of cisplatin. We modeled the cell viability responses of four different ovarian cancer cell lines (namely, OV2008, C13, A2780 and A2780-AD) following PARP and proteasome inhibition in combination using olaparib and bortezomib, respectively and analyzed each drugs' contribution to drug combination effect on cell viability. This is the first study analyzing the combined effect of these two antineoplastic agents in ovarian cancer in vitro, considering their chemosensitivity profiles. We believe that the preliminary data presented in the current in vitro study will guide further research to study this particular drug combination in more advanced experimental models of ovarian cancer including in vivo animal models. We also provided our experimental data as tables to contribute to open cancer research data to make it possible for other cancer researchers to completely reproduce the analysis we performed.

\section{MATERIALS AND METHODS}

\section{Cell Culture and Reagents}

Human ovarian cancer cell lines (OV2008, C13, A2780 and A2780-AD) were generously provided by Dr. Shelly B. Hooks, University of Georgia. These cells were maintained in RPMI1640 medium (Sigma-Aldrich) supplemented with 10\% fetal bovine serum, $5 \mathrm{mM} \mathrm{L-glutamine}$ and $5 \mathrm{mM}$ pen/strep, in humidified $5 \% \mathrm{CO}_{2}$ incubator at $37^{\circ} \mathrm{C}$. Chemoresistant cell lines C13 and A2780-AD were continuously grown with $3 \mu \mathrm{M}$ cisplatin. The cell lines have the following characteristics: OV2008 (66y, serous histotype, sequence variation: heterozygous for PIK3CA p.Glu545Lys (c.1633G>A)) (10), A2780 (age unspecified, endometrioid histotype, sequence variations: ATM p.Pro604Ser (c.1810C>T), PTEN p.Lys128_Arg130del (c.383_391del9)) $(11,12)$. All cell lines used in the present study are epithelial ovarian cancer cell lines (serous and endometrioid subtypes).

\section{Chemotherapeutic Agents}

Cisplatin was purchased from Kocak Pharma (Istanbul, Turkey). Bortezomib was purchased from LC Laboratories (Woburn, MA, USA). Olaparib was purchased from Sigma-Aldrich (St. Louis, MO, USA). Bortezomib and olaparib were dissolved in dimethyl sulfoxide (DMSO, ultra-pure grade, Amresco, VWR) and DMSO controls were included in the assays. The bortezomib stock solution was kept at $-20^{\circ} \mathrm{C}$ and the olaparib stock solution was kept at $4{ }^{\circ} \mathrm{C}$. Cisplatin was diluted in phosphate-buffered saline (PBS) and was kept at room temperature (RT) in the dark. Extensive free-thaw cycles were avoided in the storage of drugs. Olaparib concentrations used on ovarian cancer cell lines were in the 0-20 $\mu \mathrm{M}$ range, and bortezomib concentrations used were in $0-20 \mathrm{nM}$ range. Only one concentration of cisplatin was used (10 $\mu \mathrm{M})$ in triple combination treatments in the present study. The drug concentrations used in combination treatments were determined based on minimum effective drug concentrations on cell viability (i.e. $10 \mu \mathrm{M}$ for olaparib and $10 \mathrm{nM}$ for bortezomib).

\section{Cell Viability Assay}

The viability of cells after drug treatment were determined by sulphorhodamine B (SRB) colorimetric assay as previously described $(13,14)$. Briefly, cells were seeded in 96-well flat-bottom plates in $200 \mu \mathrm{l}$ media as 10,000 cells per well and allowed to adhere overnight. The cells were incubated with bortezomib and/or olaparib for $24 \mathrm{~h}$ and then, cisplatin was added, and the cells were further incubated for an additional $48 \mathrm{~h}$. Following the fixation of cells to bottom of the plate with TCA (trichloroacetic acid) solution, $200 \mu$ SRB dye solution in 1\% acetic acid was added to each well and the plates were incubated for 30 minutes using an orbital shaker. Then, washing steps were performed with $1 \%$ acetic acid four times and the plates were left to dry at a $50^{\circ} \mathrm{C}$ incubator. At the final step of SRB assay, following solubilization of dye with Tris-base solution, spectrophotometric reading (Multiskan Go, Thermo Scientific) was performed to measure absorbances at $492 \mathrm{~nm}$. Sulphorhodamine B dye was purchased from Sigma-Aldrich (St. Louis, MO, USA). All experiments were repeated at least three times (with $n \geq 3$ in each experiment).

\section{Flow Cytometry}

PE Annexin V Apoptosis Detection Kit was used to determine the effects of bortezomib and olaparib on apoptosis and necrosis in chemosensitive and chemoresistant ovarian cancer cells. The cells were plated in 12-well culture dishes and treated with bortezomib and olaparib considering the dose and time points determined in the previous cytotoxicity analyses. Following the drug incubation period, the assay was followed according to the manufacturer's instructions. Briefly, cells were harvested and washed twice with ice-cold PBS, and then resuspended in a $1 X$ binding buffer. $5 \mu$ of PE Annexin V and/or 7-AAD (7-aminoactinomycin D) were added and incubated for 15 minutes at room temperature. The cells were then analyzed within 1 hour by flow cytometry (Attune Acoustic Focusing Cytometer, Applied Biosystems). Living cells were negative for Annexin $V$ or 7-AAD, while early apoptotic cells were positive for Annexin V. Necrotic cells were counted as positive for 7-AAD, while apoptotic cells were positive for both Annexin $V$ and 7-AAD.

\section{Statistical Analysis and Data Visualization}

The data visualization and analysis were performed in R statistical programming language / environment (15-17). The follow- 
ing $\mathrm{R}$ packages were used in this study: tidyverse (a collection of R packages) (18), readxl (19), plot3D (20), magick (21), gridExtra (22), ggpubr (23) and rmarkdown $(24,25)$. The data in this study were derived from at least three independent biological repeats. Im function of base $\mathrm{R}$ was used to fit linear models using percent cell viability values (as response) and drug concentrations (as terms). Model coefficients were analyzed in order to identify the drugs' contributions to the combinatorial effect on cell viability. More detail can be found in the figure legends. Statistical comparisons in boxplots were performed with Student's $\mathrm{t}$-Test using functions from ggpubr R package (23).

\section{RESULTS}

\section{Combination of Olaparib and Bortezomib Affects Cell Via- bility in a Cell Line-Dependent Manner}

We first treated four different ovarian cancer cell lines with previously selected concentrations of olaparib and bortezomib to see how combined PARP and proteasome inhibition affects cancer cell viability. We used two chemosensitive and chemoresistant ovarian cancer cell line pairs (OV2008 - C13 and A2780 A2780-AD; the former being chemosensitive). By using the data obtained following drug treatments, we modeled cell viability (as a response) using olaparib and bortezomib concentrations as predictors. Model coefficients were used to interpret each drugs' contributions to combined cytotoxic effect.

For OV2008 chemosensitive cell line, the model coefficients for bortezomib and olaparib were -1.291658 and -1.723806 , respectively (Figure 1, upper row, first plot). This means that both drugs are associated with decreased cell viability when used in combination, although bortezomib contributes slightly to the effect of combination treatment in this chemosensitive ovarian cancer cell line. When we modeled the data for $\mathrm{C} 13$ cell line, we saw that increased cytotoxicity in the combination treatment is mostly due to olaparib, and bortezomib treatment results only in a slightly lower cell viability when used in combination with olaparib (model coefficients: -0.6809321 (bortezomib), -1.8865199 (olaparib)) (Figure 1). Therefore, it can be stated that combination of olaparib with bortezomib does not increase cytotoxicity significantly compared to olaparib alone in this chemoresistant ovarian cancer cell line.

The model coefficients were -1.083256 and -2.895892 for bortezomib and olaparib in A2780 chemosensitive cell line, respectively (Figure 1). These results indicate that the contribution of olaparib to the effect of drug combination was almost 3 times higher than that of bortezomib. Therefore, it can be pointed out that bortezomib has a slight effect on cell viability when combined with olaparib in this chemosensitive ovarian cancer cell line. For its chemoresistant subline A2780-AD, the difference in the cytotoxicity of the drugs was more dramatic (model coefficients: - 0.5706074 (bortezomib), -3.4457898 (olaparib)) (Figure 1, upper row, last plot). This shows that bortezomib has almost no increasing cytotoxic effect in combination treatment, and single olaparib treatment is already highly cytotoxic itself in this chemoresistant ovarian cancer cell line.
Percent cell viability values for treatments used in modeling are shown in bottom row of Figure 1 with corresponding $p$ values. Percent cell viability values are given in Table 1 in more detail for each drug treatment combination performed in this study to make the analysis more reproducible by other researchers.

\section{The Effect of Olaparib and Bortezomib Combination Treatment on Ovarian Cancer Cell Lines in the Presence of Cisplatin}

Next, we investigated how the effect of olaparib and bortezomib combination treatment on ovarian cancer cell lines changes when cells were also treated with cisplatin, a DNA-damaging agent used in the standard treatment of ovarian cancer. When $10 \mu \mathrm{M}$ cisplatin was applied following olaparib and/or bortezomib treatment, we observed that the cytotoxicity of bortezomib or olaparib on OV2008 cell line was low since cisplatin alone is highly cytotoxic on this chemosensitive cell line (Model coefficients: -0.70049775 (bortezomib), -0.04669829 (olaparib)) (Figure 2, upper row, first plot). Particularly, olaparib has almost zero effect on cellular viability when combined with cisplatin and bortezomib. When we considered its chemoresistant subline $\mathrm{C} 13$, both drugs had very close model coefficients (-0.9430565 (bortezomib), -0.9290382 (olaparib)), indicating that both drugs contribute almost equally to the combination treatment when used in combination with cisplatin (Figure 2). Bortezomib and olaparib decreased cell viability to a higher extent when combined with cisplatin in C13 cell line when compared to the chemosensitive OV2008 cell line.

Similar to the other chemosensitive cell line (OV2008), bortezomib and olaparib showed very slight effects on cell viability of A2780 cell line when combined with cisplatin (Model coefficients: -0.2038118 (bortezomib), -0.5011552 (olaparib)) (Figure 2). This showed that bortezomib and olaparib combination treatment does not increase cytotoxicity caused by cisplatin alone in this cell line. For its chemoresistant subline A2780-AD, the drugs' effect on cellular viability was higher (Model coefficients: -0.8512043 (bortezomib), -1.2631613 (olaparib)), indicating that combination treatment might increase cytotoxicity of cisplatin on this cell line to a certain extent and the contribution of olaparib to combinatorial effect was higher relative to that of bortezomib (Figure 2).

Percent cell viability values for treatments with cisplatin, used in modeling are shown in bottom row of Figure 2 with corresponding $\mathrm{p}$ values. Percent cell viability values are given in Table 1 in more detail for each drug treatment combination performed in this study to make the analysis more reproducible by other researchers.

Combination Treatment Results in Increased Cell Death in Chemosensitive Ovarian Cancer Cell Line OV2008

Next, we performed flow cytometry analysis using Annexin V and 7-AAD staining in chemosensitive ovarian cancer cell line OV2008 and its chemoresistant subline C13 following drug treatments. We observed that bortezomib and olaparib combination treatment resulted in an increased number of apoptotic 

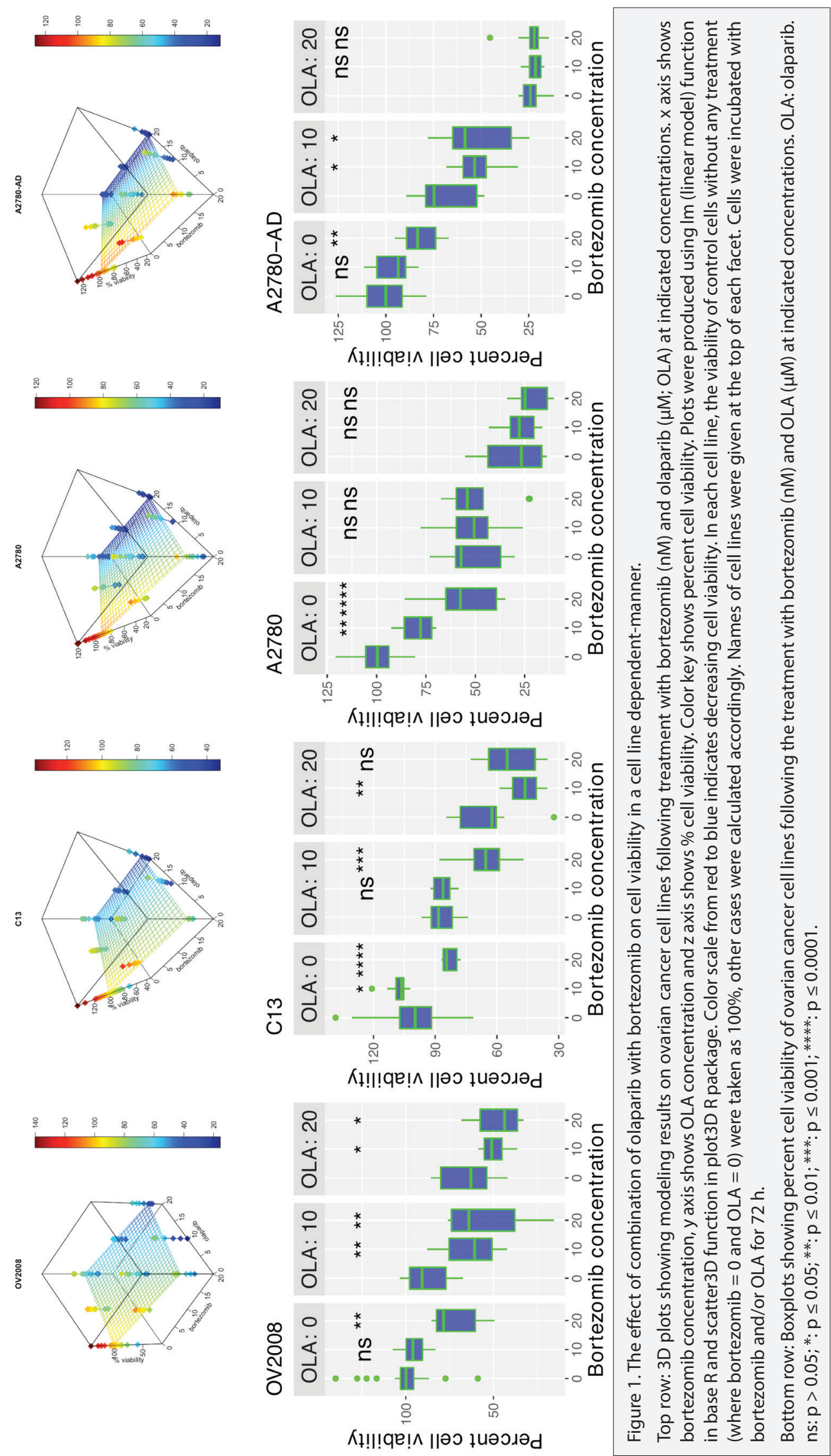
Eur J Biol 2020; 79(2): 115-123

Berkel et al. Olaparib and Bortezomib in Ovarian Cancer

Table1. Mean percent cell viability values for each drug combination treatment performed in this study for all cell lines.

\begin{tabular}{|c|c|c|c|c|}
\hline "CIS" & "BOR" & "OLA" & "Cell Line" & "Mean Viability" \\
\hline 0 & 0 & 0 & "A2780" & 99.99 \\
\hline 0 & 0 & 0 & "A2780-AD" & 99.99 \\
\hline 0 & 0 & 0 & "C13" & 99.99 \\
\hline 0 & 0 & 0 & "OV2008" & 99.99 \\
\hline 0 & 0 & 3 & "A2780" & 72.92 \\
\hline 0 & 0 & 3 & "A2780-AD" & 98.33 \\
\hline 0 & 0 & 3 & "C13" & 98.09 \\
\hline 0 & 0 & 3 & "OV2008" & 99.54 \\
\hline 0 & 0 & 5 & "A2780" & 58.75 \\
\hline 0 & 0 & 5 & "A2780-AD" & 87.65 \\
\hline 0 & 0 & 5 & "C13" & 91.09 \\
\hline 0 & 0 & 5 & "OV2008" & 102.56 \\
\hline 0 & 0 & 10 & "A2780" & 51.77 \\
\hline 0 & 0 & 10 & "A2780-AD" & 68.87 \\
\hline 0 & 0 & 10 & "C13" & 86.36 \\
\hline 0 & 0 & 10 & "OV2008" & 87.65 \\
\hline 0 & 0 & 20 & "A2780" & 29.04 \\
\hline 0 & 0 & 20 & "A2780-AD" & 23.28 \\
\hline 0 & 0 & 20 & "C13" & 65.43 \\
\hline 0 & 0 & 20 & "OV2008" & 64.35 \\
\hline 0 & 0 & 30 & "A2780" & 17.3 \\
\hline 0 & 0 & 30 & "A2780-AD" & 25.12 \\
\hline 0 & 0 & 30 & "C13" & 66.31 \\
\hline 0 & 0 & 30 & "OV2008" & 67.69 \\
\hline 0 & 0 & 40 & "A2780" & 15.36 \\
\hline 0 & 0 & 40 & "A2780-AD" & 24.54 \\
\hline 0 & 5 & 0 & "A2780" & 88.56 \\
\hline 0 & 5 & 0 & "A2780-AD" & 105.26 \\
\hline 0 & 5 & 0 & "C13" & 106.47 \\
\hline 0 & 5 & 0 & "OV2008" & 102.74 \\
\hline 0 & 10 & 0 & "A2780" & 79.45 \\
\hline 0 & 10 & 0 & "A2780-AD" & 96.37 \\
\hline 0 & 10 & 0 & "C13" & 108.14 \\
\hline 0 & 10 & 0 & "OV2008" & 94.83 \\
\hline 0 & 10 & 10 & "A2780" & 50.96 \\
\hline 0 & 10 & 10 & "A2780-AD" & 51.93 \\
\hline 0 & 10 & 10 & "C13" & 86.34 \\
\hline 0 & 10 & 10 & "OV2008" & 63.32 \\
\hline 0 & 10 & 20 & "A2780" & 27.63 \\
\hline 0 & 10 & 20 & "A2780-AD" & 21.96 \\
\hline 0 & 10 & 20 & "C13" & 46.75 \\
\hline 0 & 10 & 20 & "OV2008" & 49.46 \\
\hline 0 & 20 & 0 & "A2780" & 55.18 \\
\hline 0 & 20 & 0 & "A2780-AD" & 81.83 \\
\hline 0 & 20 & 0 & "C13" & 82.91 \\
\hline 0 & 20 & 0 & "OV2008" & 71.29 \\
\hline 0 & 20 & 10 & "A2780" & 49.37 \\
\hline 0 & 20 & 10 & "A2780-AD" & 51.32 \\
\hline 0 & 20 & 10 & "C13" & 65.36 \\
\hline 0 & 20 & 10 & "OV2008" & 54.59 \\
\hline 0 & 20 & 20 & "A2780" & 21.46 \\
\hline 0 & 20 & 20 & "A2780-AD" & 23.47 \\
\hline 0 & 20 & 20 & "C13" & 54.08 \\
\hline 0 & 20 & 20 & "OV2008" & 47.26 \\
\hline 0 & 30 & 0 & "A2780" & 30.37 \\
\hline 0 & 30 & 0 & "A2780-AD" & 38.76 \\
\hline
\end{tabular}

\begin{tabular}{|c|c|c|c|c|}
\hline 0 & 30 & 0 & "C13" & 80.89 \\
\hline 0 & 30 & 0 & "OV2008" & 54.44 \\
\hline 0 & 40 & 0 & "A2780" & 27.64 \\
\hline 0 & 40 & 0 & "A2780-AD" & 33.78 \\
\hline 0 & 40 & 0 & "C13" & 73.43 \\
\hline 0 & 40 & 0 & "OV2008" & 47.03 \\
\hline 1.5 & 0 & 0 & "A2780" & 86.13 \\
\hline 1.5 & 0 & 0 & "A2780-AD" & 97.94 \\
\hline 1.5 & 0 & 0 & "C13" & 96.34 \\
\hline 1.5 & 0 & 0 & "OV2008" & 94.08 \\
\hline 3 & 0 & 0 & "A2780" & 82.6 \\
\hline 3 & 0 & 0 & "A2780-AD" & 102.83 \\
\hline 3 & 0 & 0 & “C13" & 94.285 \\
\hline 3 & 0 & 0 & "OV2008" & 87.56 \\
\hline 5 & 0 & 0 & "A2780" & 67.2 \\
\hline 5 & 0 & 0 & "A2780-AD" & 85.78 \\
\hline 5 & 0 & 0 & "C13" & 96.3 \\
\hline 5 & 0 & 0 & "OV2008" & 85.2 \\
\hline 10 & 0 & 0 & "A2780" & 61.81 \\
\hline 10 & 0 & 0 & "A2780-AD" & 76.76 \\
\hline 10 & 0 & 0 & "C13" & 87.23 \\
\hline 10 & 0 & 0 & "OV2008" & 33.13 \\
\hline 10 & 0 & 10 & "A2780" & 57.57 \\
\hline 10 & 0 & 10 & "A2780-AD" & 51.01 \\
\hline 10 & 0 & 10 & "C13" & 81.63 \\
\hline 10 & 0 & 10 & "OV2008" & 27.57 \\
\hline 10 & 0 & 20 & "C13" & 65.77 \\
\hline 10 & 0 & 20 & "OV2008" & 25.32 \\
\hline 10 & 10 & 0 & "A2780" & 54.93 \\
\hline 10 & 10 & 0 & "A2780-AD" & 57.87 \\
\hline 10 & 10 & 0 & "C13" & 93.11 \\
\hline 10 & 10 & 0 & "OV2008" & 31.9 \\
\hline 10 & 10 & 10 & "A2780" & 52.93 \\
\hline 10 & 10 & 10 & "A2780-AD" & 50.99 \\
\hline 10 & 10 & 10 & "C13" & 77.88 \\
\hline 10 & 10 & 10 & "OV2008" & 26.77 \\
\hline 10 & 10 & 20 & "C13" & 48.4 \\
\hline 10 & 10 & 20 & "OV2008" & 34.18 \\
\hline 10 & 20 & 0 & "A2780" & 60.95 \\
\hline 10 & 20 & 0 & "A2780-AD" & 49.5 \\
\hline 10 & 20 & 0 & "C13" & 53.05 \\
\hline 10 & 20 & 0 & "OV2008" & 9.01 \\
\hline 10 & 20 & 10 & "A2780" & 51.8 \\
\hline 10 & 20 & 10 & "A2780-AD" & 47.18 \\
\hline 10 & 20 & 10 & "C13" & 58.95 \\
\hline 10 & 20 & 10 & "OV2008" & 10.27 \\
\hline 10 & 20 & 20 & "C $13 "$ & 65.21 \\
\hline 10 & 20 & 20 & "OV2008" & 19.92 \\
\hline 20 & 0 & 0 & "A2780" & 51.55 \\
\hline 20 & 0 & 0 & "A2780-AD" & 77.24 \\
\hline 20 & 0 & 0 & "C13" & 88.26 \\
\hline 20 & 0 & 0 & "OV2008" & 24.93 \\
\hline 30 & 0 & 0 & "A2780" & 30.8 \\
\hline 30 & 0 & 0 & "A2780-AD" & 27.81 \\
\hline 30 & 0 & 0 & "C $13 "$ & 72.67 \\
\hline 30 & 0 & 0 & "OV2008" & 7.19 \\
\hline 40 & 0 & 0 & "A2780" & 19.84 \\
\hline 40 & 0 & 0 & "A2780-AD" & 23.66 \\
\hline 40 & 0 & 0 & "C13" & 67.34 \\
\hline 40 & 0 & 0 & "OV2008" & 11.09 \\
\hline
\end{tabular}



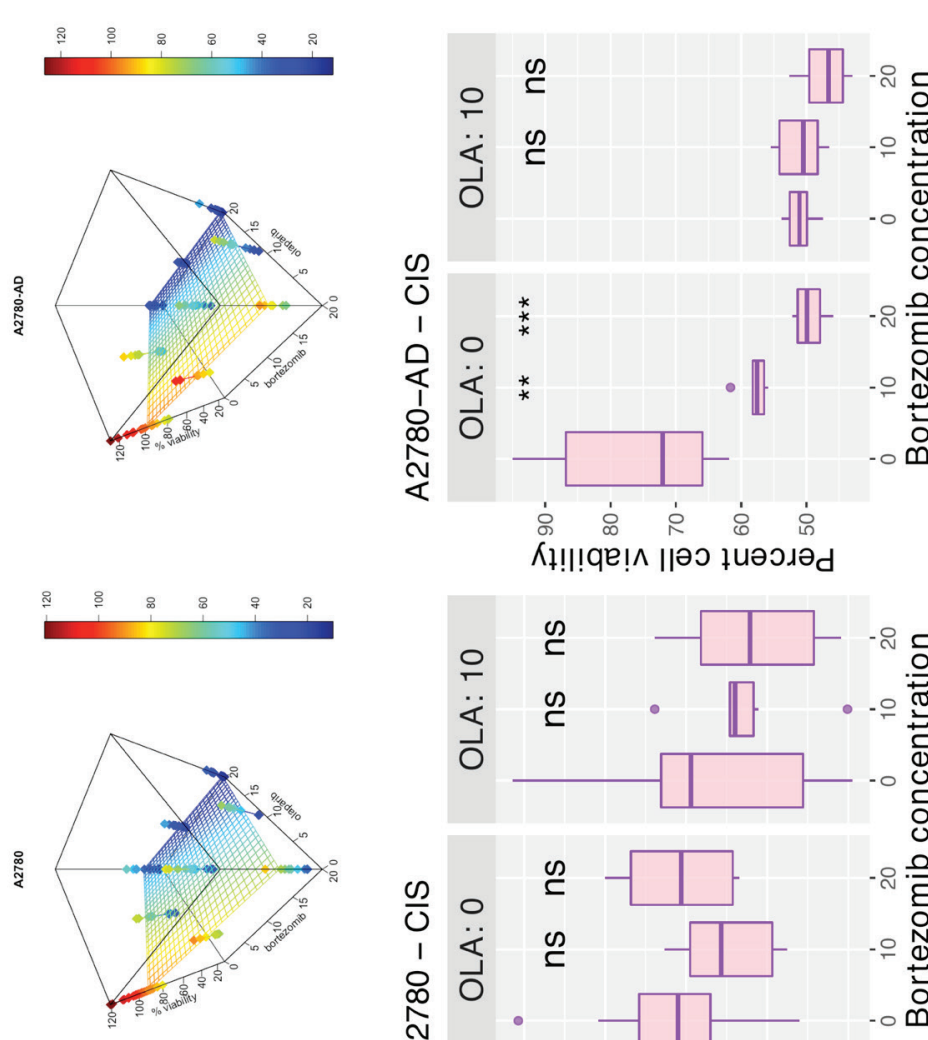

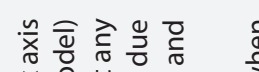

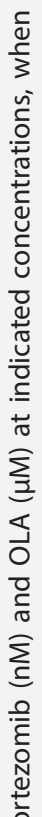
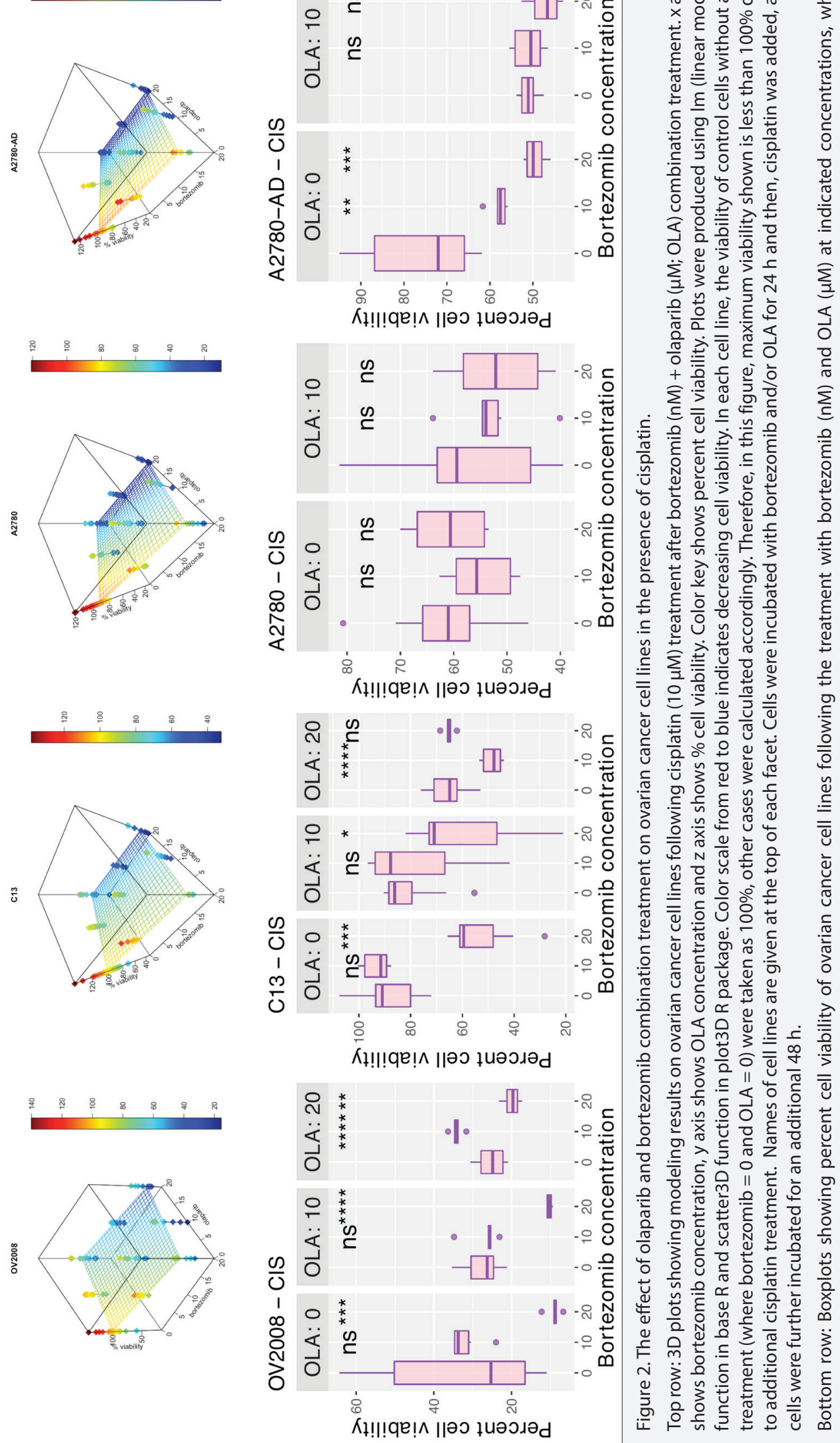

$\dot{8}$
$\dot{8}$
$\dot{0}$
$\mathrm{~V} 1$

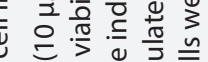

粶

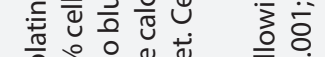

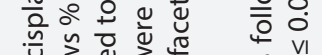

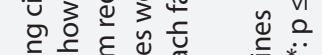

ज实施

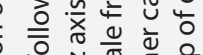

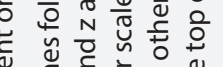

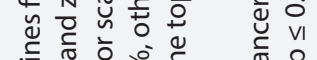

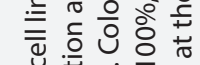

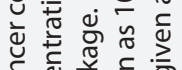

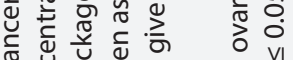

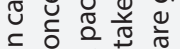

등 웅

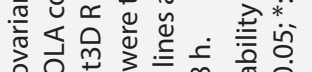

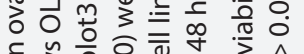

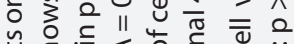

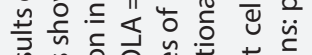

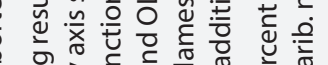

कृ

政

을 을

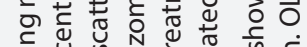

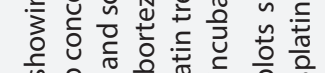

प্ّ

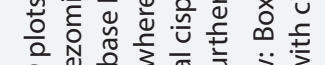

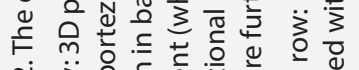

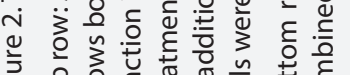

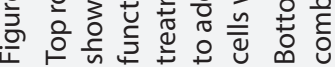



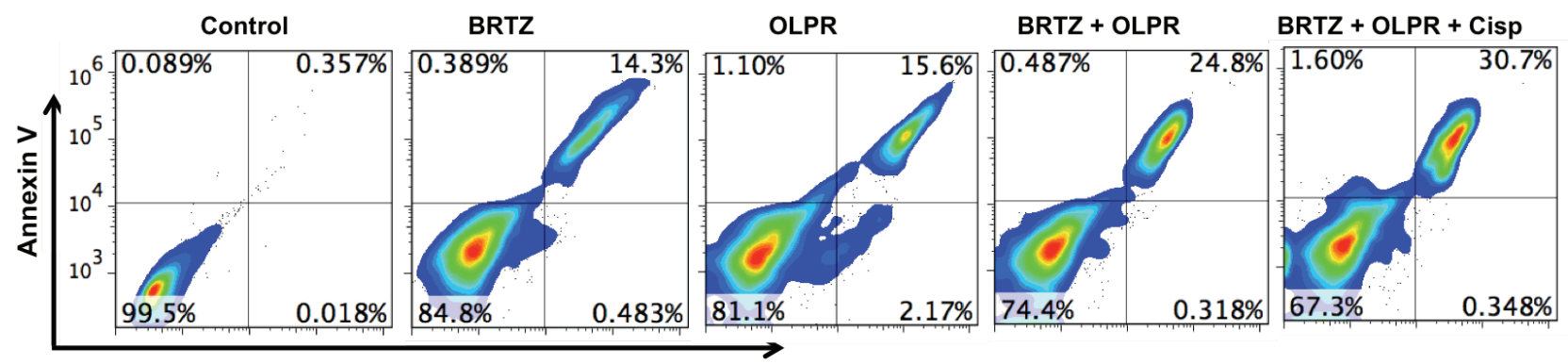

7-AAD

B) C13
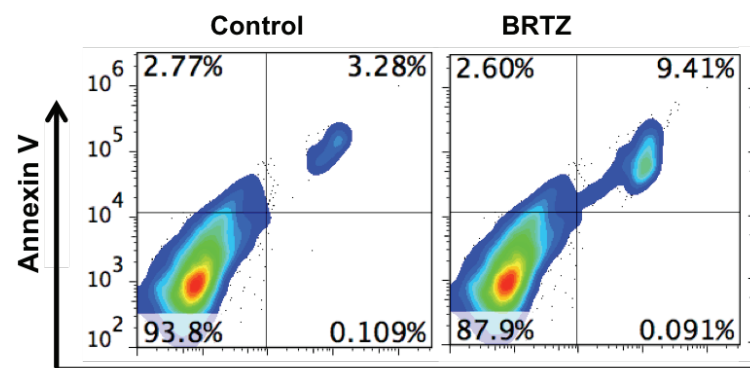

OLPR

BRTZ + OLPR

BRTZ + OLPR + Cisp
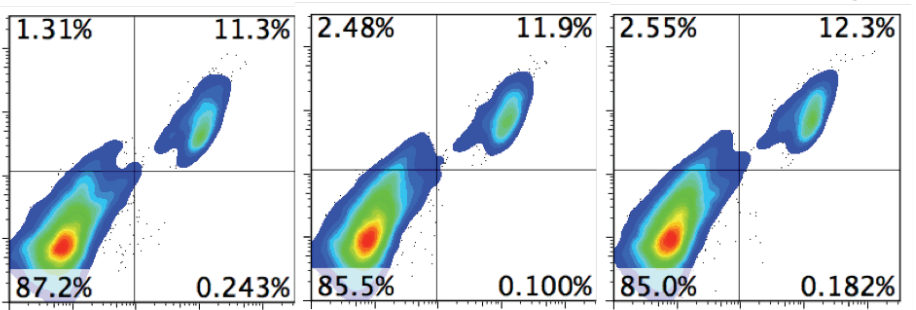

7-AAD

Figure 3. Combination treatment results in increased cell death in chemosensitive ovarian cancer cell line OV2008.

Flow cytometry analysis with Annexin $V$ and 7-AAD to study the effect of combination treatment on chemosensitive and chemoresistant ovarian cancer cell lines (OV2008 (A) and C13 (B), respectively). X-axis shows the number of 7-AAD-positive cells and y- axis shows the number of Annexin V-positive cells. Drug treatment conditions are given at the top of each facet. Percentage of cells in each region were given in corresponding corner. Upper right region with high Annexin V and 7-AAD values shows dead cells. BRTZ: bortezomib, OLPR / OLA: olaparib, Cisp: cisplatin.

cells in OV2008 cell line compared to drugs alone (bortezomib only or olaparib only) (Figure 3A). However, in chemoresistant cell line $\mathrm{C} 13$, we did not see any significant increase in apoptotic cells relative to drugs alone. Similar to previous experiments, the effect of cisplatin on the chemosensitive cell line OV2008 was higher compared to chemoresistant cell line C13. When we combined cisplatin with bortezomib and olaparib, we observed an increase in cell death in OV2008 cell line compared to bortezomib and olaparib combination treatment. However, this increased cytotoxicity in triple drug treatments might be due to only cisplatin since we did not perform cisplatin only treatments for the flow cytometry experiments. In C13 cell line, the bortezomib and olaparib combination did not further increase cell death relative to drugs alone. The addition of cisplatin did not further increase apoptotic cell death compared to olaparib and bortezomib combination treatment in this chemoresistant cell line (Figure 3).

\section{DISCUSSION}

In the current study, we investigated the effect of combinatorial inhibition of PARP and proteasome by olaparib and bortezomib, on the viability of chemosensitive or chemoresistant ovarian cancer cells. Olaparib is currently used in the clinic for a certain group of ovarian cancer patients including patients with recurrent platinum-sensitive disease with or without a BRCA mutation (4). However, bortezomib is not approved for the treatment of ovarian cancer patients (but used in some other cancer types), though multiple studies showed its efficacy when used in combination with other strategies in ovarian cancer (26-28). To our knowledge, there is no report showing the effect of combinatorial inhibition of proteasome and PARP by bortezomib and olaparib, respectively on ovarian cancer cell viability. Since most ovarian cancer patients experience chemoresistance to cisplatin following several rounds of chemotherapy, novel treatment strategies such as in the present study are urgently needed to be investigated and developed in order to increase the survival of ovarian cancer patients (29).

In this study, we observed that combination treatment is mostly effective on chemosensitive ovarian cancer cell lines (OV2008, A2780). However, with chemoresistant cell lines (C13, A2780-AD), the effect of bortezomib is minimal compared to that of olaparib, in combination. Therefore, it can be stated that combination of olaparib with bortezomib might result in higher therapeutic efficacy in chemosensitive ovarian cancer compared to drugs alone. Also, the addition of 
bortezomib to olaparib treatment might only lead to a slight increase in cytotoxicity in chemoresistant ovarian cancer cells.

When the bortezomib and olaparib combination treatment is applied with cisplatin, we see that combination treatment does not significantly decrease cell viability in chemosensitive ovarian cancer cell lines, since cisplatin treatment alone is already highly effective itself. In OV2008 chemosensitive cell line, bortezomib might decrease cell viability to a certain extent when applied with cisplatin; however, olaparib treatment does not contribute to cytotoxicity in this case. In A2780 chemosensitive cell line, both bortezomib and olaparib do not significantly affect the cellular viability when combined with cisplatin.

In $\mathrm{C} 13$ chemoresistant cell line, both bortezomib and olaparib further decrease cell viability when combined with cisplatin. Similarly, in the other chemoresistant cell line, A2780-AD, both drug treatments might lead to a lower cell viability when used together with cisplatin. Therefore, it can be pointed out that in the presence of cisplatin, combination treatment is mostly effective on chemoresistant cell lines (C13, A2780-AD) when compared to drugs alone.

In flow cytometry data, we observed that bortezomib and olaparib combination treatment leads to increased apoptotic cell death relative to drugs alone in OV2008 chemosensitive cell line. We also saw that the combination of bortezomib and olaparib with cisplatin resulted in increased cell death compared to bortezomib and olaparib combination treatment in this cell line. Considering previous data, this should be mostly due to cisplatin which is already highly effective on chemosensitive ovarian cancer cell lines. Therefore, it can be stated that olaparib and bortezomib combination treatment does not further increase cytotoxicity compared to cisplatin alone in OV2008 chemosensitive cell line; however, olaparib and bortezomib combination increases cell death compared to single agent treatments. This result points to the fact that patients who respond to olaparib treatment but not to cisplatin might benefit from bortezomib + olaparib combination compared to olaparib only treatment. However, for patients who respond to cisplatin treatment, bortezomib + olaparib treatment might not be a preferable clinical option. A more concrete inference based on this data requires in vivo experiments performed in mouse models. Considering the cellular and inter-patient heterogeneity in ovarian cancer (most generally in cancer), personalized treatment alternatives should be developed for patients with various chemosensitivity profiles, since chemosensitivity characteristics of cancer cells also influence their response to treatment as also shown in the present study (30-33). In C13 cell line, we saw that combination treatment did not result in increased cell death compared to drugs alone and this is in line with previous experiments. In previous experiments, we saw that in the presence of cisplatin, the effect of bortezomib and olaparib combination treatment was higher in C13 cell line compared to OV2008 cell line; but, in flow cytometry, we saw that cisplatin addition did not increase cell death compared to bortezomib and olaparib treatment.

\section{CONCLUSION}

In conclusion, our data shows that combination treatment is particularly effective on chemosensitive ovarian cancer cell lines in the absence of cisplatin. However, when combined with cisplatin, olaparib and bortezomib combination treatment shows its effect especially on chemoresistant ovarian cancer cell lines. As a result, combinatorial inhibition of PARP and proteasome by olaparib and bortezomib can be tested in vivo on chemoresistant ovarian cancer which are also treated with cisplatin to overcome cisplatin resistance in these ovarian cancer cell lines. Since the data provided in the current study were obtained using in vitro cell line models, in vivo studies performed on animal models should be required in the future to make stronger inferences about the effect of combinatorial treatment with bortezomib and olaparib on ovarian cancer.

Peer-review: Externally peer-reviewed.

Author Contributions: Conception/Design of study: B.K., M.U., E.C.; Data Acquisition: C.B., E.Y., E.C.; Data Analysis/Interpretation: C.B., E.C.; Drafting Manuscript: C.B.; Critical Revision of Manuscript: E.C.; Final Approval and Accountability: C.B., E.C.; Supervision: E.C.

Conflict of Interest: The authors declare that they have no conflicts of interest to disclose.

Financial Disclosure: This work was supported by 2209-A program of Scientific and Technological Research Council of Turkey (TUBITAK) (1919B011700125). C.B. is funded by TUBITAK 2211-E graduate student program.

Acknowledgement: Authors declare no conflicts of interest. Authors would like to thank Master's student Muhsine OZEN for her support.

\section{REFERENCES}

1. Bray F, Ferlay J, Soerjomataram I, Siegel RL, Torre LA, Jemal A. Global cancer statistics 2018: GLOBOCAN estimates of incidence and mortality worldwide for 36 cancers in 185 countries. CA Cancer J Clin 2018; 68(6): 394-424.

2. Berkel C, Cacan E. In silico analysis of DYNLL1 expression in ovarian cancer chemoresistance. Cell Biol Int 2020; 44(8): 1598-605.

3. Ali MW, Cacan E, Liu Y, Pierce JY, Creasman WT, Murph MM, Govindarajan R, Eblen ST, Greer SF, Hooks SB. Transcriptional suppression, DNA methylation, and histone deacetylation of the regulator of G-protein signaling 10 (RGS10) gene in ovarian cancer cells. PLoS One 2013; 8(3): e60185.

4. Cacan E, Ali MW, Boyd NH, Hooks SB, Greer SF. Inhibition of HDAC1 and DNMT1 modulate RGS10 expression and decrease ovarian cancer chemoresistance. PLoS One 2014; 9(1): e87455.

5. European Medicines Agency. Lynparza summary of product characteristics, 2018; Available at: http://ec.europa.eu/health/documents/ community-register/2018/20180508140545/anx_140545_en.pdf.

6. Morales J, Li L, Fattah FJ, Dong Y, Bey EA, Patel M, et al. Review of poly (ADP-ribose) polymerase (PARP) mechanisms of action and rationale for targeting in cancer and other diseases. Crit Rev Eukaryot Gene Expr 2014; 24(1): 15-28. 
7. Vescarelli E, Gerini G, Megiorni F, Anastasiadou E, Pontecorvi P, Solito $L$, et al. MiR-200c sensitizes Olaparib-resistant ovarian cancer cells by targeting Neuropilin 1. J Exp Clin Cancer Res 2020; 39(1): 3.

8. Wang L, Shi C, Wright FA, Guo D, Wang X, Wang D, et al. Multifunctional telodendrimer nanocarriers restore synergy of bortezomib and doxorubicin in ovarian cancer treatment. Cancer Res 2017; 77(12): 3293-305.

9. Cacan E, Spring AM, Kumari A, Greer SF, Garnett-Benson C. Combination treatment with sublethal ionizing radiation and the proteasome inhibitor, bortezomib, enhances death-receptor mediated apoptosis and anti-tumor immune attack. Int J Mol Sci 2015; 16(12): 30405-21.

10. Takenaka M, Saito M, Iwakawa R, Yanaihara N, Saito M, Kato M, et al. Profiling of actionable gene alterations in ovarian cancer by targeted deep sequencing. Int J Oncol 2015; 46(6): 2389-98.

11. Anglesio MS, Wiegand KC, Melnyk N, Chow C, Salamanca C, Prentice LM, et al. Type-specific cell line models for type-specific ovarian cancer research. PLoS One 2013; 8(9): e72162.

12. Beaufort CM, Helmijr JC, Piskorz AM, Hoogstraat M, Ruigrok-Ritstier K, Besselink N, et al. Ovarian cancer cell line panel (OCCP): clinical importance of in vitro morphological subtypes. PLoS One 2014; 9(9): e103988.

13. Vichai V, Kirtikara K. Sulforhodamine B colorimetric assay for cytotoxicity screening. Nat Protoc 2006; 1(3): 1112-6.

14. Skehan P, Storeng R, Scudiero D, Monks A, McMahon J, Vistica D, et al. New colorimetric cytotoxicity assay for anticancer-drug screening. J Natl Cancer Inst 1990; 82(13): 1107-112.

15. R Core Team. R: A language and environment for statistical computing. R Foundation for Statistical Computing, 2018; Vienna, Austria. URL https://www.R-project.org/.

16. Grolemund G, Wickham H. R for data science, 2016; 1st edition. California: O'Reilly.

17. Holmes S, Huber W. Modern statistics for modern biology, 2019; 1st edition. Cambridge: Cambridge University Press.

18. Wickham $\mathrm{H}$. tidyverse: Easily Install and Load the 'Tidyverse'. $\mathrm{R}$ package version, 2017, 1.2.1. https://CRAN.R-project.org/package=tidyverse

19. Wickham $\mathrm{H}$ and Bryan J. readxl: Read Excel Files. R package version 1.3.1, 2019. https://CRAN.R-project.org/package=readxl

20. Soetaert K. plot3D: Plotting Multi-Dimensional Data. R package version 1.3, 2019. https://CRAN.R-project.org/package=plot3D
21. Ooms J. magick: Advanced graphics and image-processing in R. $\mathrm{R}$ package version 2.4.0, 2020. https://CRAN.R-project.org/package $=$ magick

22. Auguie B. gridExtra: Miscellaneous functions for "Grid" graphics. R package version 2.3, 2017. https://CRAN.R-project.org/package $=$ gridExtra

23. Kassambara A. ggpubr: 'ggplot2' Based Publication Ready Plots. R package version 0.4.0, 2020. https://CRAN.R-project.org/package $=$ ggpubr

24. Xie Y, Allaire JJ, Grolemund G. R Markdown: The Definitive Guide. Chapman and Hall/CRC, 2018. ISBN 9781138359338. URL https:// bookdown.org/yihui/rmarkdown.

25. Xie Y, Dervieux C, Riederer E. R Markdown Cookbook. Chapman and Hall/CRC, 2020. ISBN 9780367563837. URL https://bookdown. org/yihui/rmarkdown-cookbook.

26. He YJ, Meghani K, Caron MC, Yang C, Ronato DA, Bian J, et al. DYNLL1 binds to MRE1 1 to limit DNA end resection in BRCA1-deficient cells. Nature 2018; 563(7732): 522-6.

27. Janyst K, Janyst M, Siernicka M, Lasek W. Synergistic antitumor effects of histone deacetylase inhibitor scriptaid and bortezomib against ovarian cancer cells. Oncol Rep 2018; 39(4): 1999-2005.

28. Wang L, Shi C, Wright FA, Guo D, Wang X, Wang D, et al. Multifunctional telodendrimer nanocarriers restore synergy of bortezomib and doxorubicin in ovarian cancer treatment. Cancer Res 2017; 77(12): 3293-305.

29. Berkel C, Cacan E. GAB2 and GAB3 are expressed in a tumor stage-, grade- and histotype-dependent manner and are associated with shorter progression-free survival in ovarian cancer. J Cell Commun Signal 2020;10.1007/s12079-020-00582-3.

30. Berkel C, Cacan E. Single-cell epigenomics in cancer research. Biomed J Sci\&Tech Res 2019; 21(3).

31. Kroeger PT, Jr, \& Drapkin R. Pathogenesis and heterogeneity of ovarian cancer. Curr Opin Obstet Gynecol 2017; 29(1): 26-34.

32. Winterhoff BJ, Maile M, Mitra AK, Sebe A, Bazzaro M, Geller MA, et al. Single cell sequencing reveals heterogeneity within ovarian cancer epithelium and cancer associated stromal cells. Gynecol Oncol 2017; 144(3): 598-606.

33. Shih AJ, Menzin A, Whyte J, Lovecchio J, Liew A, Khalili $H$, et al. Identification of grade and origin specific cell populations in serous epithelial ovarian cancer by single cell RNA-seq. PLoS One 2018; 13(11): e0206785. 